\title{
Appareil
}

$20 \mid 2018$

Arts contemporains et patrimoine

\section{À propos de l'hospitalité : institution et inconditionnalité}

Daniel Payot

\section{(2) OpenEdition}

\section{Journals}

Édition électronique

URL : http://journals.openedition.org/appareil/2203

DOI : 10.4000/appareil.2203

ISSN : 2101-0714

Éditeur

MSH Paris Nord

\section{Référence électronique}

Daniel Payot, «À propos de l'hospitalité : institution et inconditionnalité », Appareil [En ligne], 20 | 2018, mis en ligne le 26 octobre 2018, consulté le 30 juillet 2020. URL : http://journals.openedition.org/ appareil/2203; DOI : https://doi.org/10.4000/appareil.2203

Ce document a été généré automatiquement le 30 juillet 2020.

\section{(c) (i) (3)}

Appareil est mis à disposition selon les termes de la Licence Creative Commons Attribution - Pas d'Utilisation Commerciale - Pas de Modification 4.0 International. 


\title{
À propos de l'hospitalité : institution et inconditionnalité
}

\author{
Daniel Payot
}

\section{NOTE DE L'AUTEUR}

Cet article issu d'une conférence prononcée à Tonnerre le 19 septembre 2015 est originellement paru dans la rubrique Galerie d'Appareil en 2016. Il a été revu par l'auteur dans le cadre de sa mobilisation pour ce numéro.

\section{À Jean-Louis Déotte}

1 Dans sa somme publiée en 1969 sous le titre Le Vocabulaire des institutions indoeuropéennes $^{1}$, le linguiste Émile Benveniste remarque, comme bien d'autres, que le mot latin hostis, qui en français a donné « hôte » et qui, à ce titre, appartient au vocabulaire de l'accueil, de la bienveillance invitante, de l'hospitalité, a aussi donné "hostile», « hostilité », une série de termes qui désigne l'ennemi. Il résume ainsi la raison que l'on donne le plus souvent à cette ambivalence :

Pour expliquer le rapport entre «hôte » et « ennemi», on admet en général que

l'un et l'autre dérivent du sens de « étranger » qui est encore attesté en latin; d'où

« étranger favorable - hôte » et « étranger hostile - ennemi ${ }^{2}$ ».

2 Mais cette explication ne convainc pas Benveniste : l'enquête qu'il mène patiemment autour du mot hostis le conduit principalement, non pas à la notion d'hostilité, mais à l'idée d'une équivalence, d'une égalisation, plus précisément à l'idée d'une égalisation par compensation. Rappelant la différence existant entre le peregrinus, l'étranger résidant dans un autre pays, et l'hostis, à qui les Romains reconnaissaient des droits égaux aux leurs, il voit dans le «lien d'égalité et de réciprocité » ainsi établi « ce qui peut conduire à la notion précise d'hospitalité »: "hostis signifiera “celui qui est en relations de compensation"; ce qui, ajoute Benveniste, est bien le fondement de l'institution d'hospitalité ${ }^{3}$. 
La suite du texte est une référence aux travaux de Marcel Mauss sur le don et le contredon, le «potlatch » :

L'hospitalité s'éclaire par référence au potlatch dont elle est une forme atténuée. Elle est fondée sur l'idée qu'un homme est lié à un autre (hostis a toujours une valeur réciproque) par l'obligation de compenser une certaine prestation dont il a été bénéficiaire ${ }^{4}$.

Puis Benveniste poursuit par des considérations sur le grec xénos, terme dans lequel il repère une histoire similaire à celle de l'hostis latin :

La même institution existe dans le monde grec sous un autre nom : xénos indique les relations du même type entre hommes liés par un pacte qui implique des obligations précises s'étendant aussi aux descendants. La xenía [...] comporte échange de dons entre les contractants qui déclarent leur intention de lier leurs descendants par ce pacte ${ }^{5}$.

3 L'hospitalité apparaît ainsi comme une procédure institutionnellement réglée, selon laquelle l'accueil de l'étranger et l'octroi de droits à ce dernier sont indissociables d'un contrat ou pacte ; cela, au préalable, suppose l'existence d'une instance du " propre ", de l'« autochtone » ou de l'originaire, instance précisément susceptible d'accorder par convention à l'autre, l'étranger, le non-identique, un droit qu'il n'avait pas d'abord.

Dans l'indication de Benveniste selon laquelle le «fondement de l'institution d'hospitalité » est le fait d'être " en relations de compensation », ce dernier terme est particulièrement significatif : il y a quelque chose à compenser, parce que l'octroi du droit, d'une certaine égalité ou équivalence, vient lui-même corriger un déséquilibre initial. La référence au potlatch indique que ce déséquilibre n'est pas forcément hiérarchique : il n'inclut pas l'idée de la supériorité de l'un sur l'autre, seulement celle de la différence entre l'espace de l'identité et l'espace de ce qui lui est extérieur, autre. Mais cela suffit à suggérer aussi la possibilité d'un lien structurel entre hospitalité et pouvoir : paradoxalement, le don de l'hospitalité pourrait bien être l'apanage de ceux qui, assurés dans leur identité, voire dans leur domination, prouvent ainsi leur pouvoir dans et par le geste même qui pourrait sembler aux antipodes du pouvoir, le geste de donner. Nous sommes là, d'emblée, dans une certaine ambivalence. La dimension du don situe la notion d'hospitalité du côté de la générosité gratuite, de l'accueil sans réserve, mais la dimension de l'octroi la place du côté du pouvoir ; entre les deux (mais y a-t-il un "entre-deux ", là est bien la question), on note l'importance du schème institutionnel, juridique, du contrat, du pacte, de la convention, de la xenía.

5 La littérature européenne - c'est là l'hypothèse que je voudrais esquisser - , a été singulièrement attentive à de telles ambivalences, quand elle s'est intéressée à la question de l'hospitalité.

6 C'est le cas, me semble-t-il, dès le début, je veux dire chez Homère. L'Odyssée pourrait être interprétée comme un texte portant principalement sur le problème de l'hospitalité, dans son ensemble et, particulièrement, dans son cœur : les chants VI à XIII qui suivent le voyage de Télémaque et précèdent le récit du retour d'Ulysse à Ithaque.

7 Dans l'avant-dernier épisode de son périple, Ulysse a été jeté par les flots, seul et nu, sur un rivage inconnu. Athéna inspire en rêve à Nausicaa, la fille du roi de ce territoire, l'idée d'aller au rivage, puis elle fait en sorte qu'Ulysse la voie et qu'une rencontre ait lieu entre $e^{6}$. Ulysse prend la parole le premier; sans dire son nom, il raconte brièvement ses malheurs, invoque les dieux et demande protection. La réponse de Nausicaa est, elle aussi, une référence à la divinité : 
Tu sais bien, étranger [...] que Zeus, de son Olympe, répartit le bonheur aux vilains comme aux nobles, ce qu'il veut pour chacun : s'il t'a donné ces maux, il faut bien les subir. Mais puisque te voilà en notre ville et terre, ne crains pas de manquer ni d'habits ni de rien que l'on doive accorder, en pareille rencontre, au pauvre suppliant. Vers le bourg, je serai ton guide et te dirai le nom de notre peuple [...]. C'est à nos Phéaciens qu'est la ville et sa terre, du fier Alkinoos, je suis la fille, du roi qui tient en main la force et la puissance de cette Phéacie ${ }^{7}$.

8 Avant de rejoindre le manoir d'Alkinoos, Ulysse adresse à Athéna une prière : «Fais que les Phéaciens m'accueillent en ami et me soient pitoyables! » On remarque ici que le don par Nausicaa du nom du peuple est ce qui permet à Ulysse de prier : le don du nom est un signe d'hospitalité; il y a de ce point de vue, dans l'épisode raconté, une dissymétrie : Ulysse n'a toujours pas dévoilé son nom, il ne le fera que plus tard, quand Alkinoos le lui demandera expressément; en revanche, Nausicaa, sans donner son propre nom, a dévoilé celui de son peuple et celui de son père, c'est-à-dire des deux puissances hospitalières.

9 Nausicaa l'ayant précédé, Ulysse entre seul dans la ville, rapidement assisté par Athéna qui, déguisée en petite fille se présentant comme enfant d'Alkinoos, le guide jusqu'à la maison du roi. Au cours du trajet, elle lui dit ceci, qui peut paraître étrange :

Suis-moi sans parler; je te montre la route; ne regarde personne et ne demande rien. Les étrangers ici reçoivent peu d'accueil ; à qui vient du dehors, on ne fait pas grand fête ni même d'amitiés; nous mettons nos espoirs en nos croiseurs rapides; car l'Ébranleur du sol [Poséidon] a concédé le grand abîme à nos passeurs : nos vaisseaux sont plus prompts que l'aile ou la pensée ${ }^{8}$.

10 Ces paroles sont étranges, parce qu'elles sont démenties par l'accueil effectif, d'abord de Nausicaa sur le rivage, ensuite d'Alkinoos au manoir, mais aussi parce qu'elles semblent mettre en opposition l'accueil de l'étranger et la rapidité des vaisseaux, comme s'il s'agissait de dire que le bien, le bonheur, le positif en général ne pouvait pas être reçu, ne pouvait pas venir unilatéralement de l'extérieur, en particulier de l'étranger, mais ne pouvait qu'être gagné après une traversée, un passage des abîmes, une conquête. En soi, dans son événement immédiat, la venue, l'arrivée de l'étranger est suspecte - tant qu'elle ne donne pas lieu à un rituel, un pacte quelconque qui en fixe les termes et en supprime précisément l'immédiateté ; tant que la venue ne donne pas lieu à la médiation d'une xenía.

11 Cette xenía, logiquement, se met en place tout de suite après. Ulysse est très bien reçu au palais, selon un rituel qui, bien sûr, comporte de nombreuses offrandes à Zeus.

À la fin du festin, Alkinoos prononce entre autres ces paroles :

Je veux qu'en ce manoir on fête l'étranger [...] Mais peut-être est-ce un dieu, qui nous descend du ciel pour un nouveau dessein que les dieux ont sur nous : ne les vîmes-nous pas, cent fois dans le passé, à nos yeux apparaître ? Quand nous faisons pour eux nos fêtes d'hécatombes, ils viennent au festin s'asseoir à nos côtés, aux mêmes bancs que nous; sur le chemin désert, s'ils croisent l'un des nôtres, ils ne se cachent point : nous sommes de leur sang'.

Cette déclaration est intéressante, dans la mesure où elle associe l'arrivée de l'étranger et l'apparition des dieux. Cela ne signifie pas vraiment que l'étranger est lui-même divinisé ou confondu avec un dieu ; et dans sa réponse, Ulysse prendra soin de dissocier très explicitement sa propre venue de celle des dieux, sa propre personne de celle d'un dieu. Mais elle renforce une dimension qui est omniprésente dans la problématique homérique de l'hospitalité : l'étranger est accueilli dans la mesure où sa venue est une décision des dieux, se faisant sous leur égide, égide attestée par un rituel, festin et 
parole, qui tient lieu de pacte. La relation, si l'on veut, est contractuelle sans être jamais duelle: l'hospitalité est une affaire de tiers: l'accueillant, l'accueilli et le dieu qui envoie, garantit, parfois aussi apparaît lui-même. Le festin, le rite mettent en place cette xenía, ce contrat, cette institutionnalisation de l'hospitalité dans laquelle nous reconnaissons donc les deux éléments que nous distinguions chez Benveniste : le don et le pacte.

Dernier point à propos de l'Odyssée : l'accueil d'Ulysse par les Phéaciens a aussi pour conséquence le récit d'Ulysse. Alkinoos lui demande de raconter ce qui lui est arrivé avant son échouage sur son rivage. Le récit d'Ulysse, qui forme la trame centrale de l' Odyssée tout entière, est d'une certaine manière ce qu'il offre en compensation de son accueil, de sa protection et de la promesse d'être conduit jusqu'à Ithaque. Or ces récits ont eux-mêmes trait à l'hospitalité ou à son défaut. Ce sont des histoires de manquements explicites à l'hospitalité, c'est-à-dire aux dieux, dans le cas des Lotophages, du Cyclope et des Lestrygons; ce sont des histoires d'hospitalités ambivalentes dans le cas de Circé et de Calypso.

Si l'Odyssée est par hypothèse un texte sur l'hospitalité, on pourrait lire Le Château de Kafka comme une Odyssée inversée et évidée. D’une certaine manière, K. arrive au village comme Ulysse chez les Phéaciens. Mais tout se passe comme si le schème de l'hospitalité se trouvait, chez Kafka, mis à l'envers. K. ne s'échoue pas, il est invité au château : la convention, le pacte a lieu d'emblée, avant même que le texte ne débute, sous la forme d'une convocation à venir travailler en tant qu'arpenteur. Mais tout ce qui suit montre que ce pacte n'est précisément pas une xenía, que la réciprocité, l'équivalence, le don et le contre-don ne fonctionnent pas. En ce sens Le Château est un grand texte sur l'absence d'hospitalité; tout ce que K. vit dans le village est une succession d'épisodes au cours lesquels il est soit explicitement rejeté comme étranger, comme intrus, soit cantonné dans un espace instable, perpétuellement provisoire, perpétuellement insatisfaisant. Mais même cette relégation n'est pas absolument univoque.

16 L'une des premières paroles qu'entend $\mathrm{K}$., au chapitre 1 , de la part d'un habitant du village, est la suivante: « Vous êtes sans doute surpris [...] de notre peu d'hospitalité. Mais l'hospitalité n'est pas d'usage chez nous, nous n'avons pas besoin d'hôtes ${ }^{10}$. » Cette déclaration ressemble à celle qu'Athéna prêtait à l'enfant d'Alkinoos, mais elle a, dans ce contexte, un sens bien différent : le refus de l'hospitalité n'a pas ici de contraire, rien d'autre ne nourrit l'espoir du peuple, et il est absolu. L'accueil n'est pas refusé parce qu'il serait immédiat, parce qu'il précéderait le pacte, il est refusé définitivement: «nous n'avons pas besoin d'hôtes ", pas besoin d'étrangers.

17 Cette position est confirmée tout au long du texte, avec quelques nuances qui renforcent en fait le dispositif d'ensemble: ainsi, au long des chapitres 6 à 8 , K. bénéficie d'une forme d'attention de la part de la famille d'Amalia, Olga et Barnabé, mais on comprend vite que cette famille est elle-même en position d'exclusion, comme s'il n'y avait plus d'accueil possible qu'entre exclus, et pas entre autochtones et étrangers, entre identité et altérité, entre Même et Autre.

18 La dimension générale du refus d'hospitalité dans le texte de Kafka est à mettre en relation avec la distance infranchissable sans cesse évoquée qui sépare le village du château. Cette séparation détermine l'espace de tous les déplacements et de toutes les relations, comme en creux: tout ce qui se passe réellement confirme le défaut de la seule instance qui, seule identique et puissante, pourrait conclure de vraies 
conventions, être partenaire et garante d'une vraie xenía. La forme du contrat est omniprésente, tellement présente que tout ce qui n'est pas elle se trouve dévalorisé ; mais en même temps, elle interdit toute détermination d'une teneur contractuelle : tout renvoie ultimement à l'alliance du village et du château, comme tout dans l'Odyssée renvoyait aux relations entre les humains et les dieux. Mais chez Kafka, cette alliance n'a pas de contenu, et à sa place s'imposent le silence incompréhensible et l'éloignement incommensurable du château. D'où l'ambivalence fondatrice qui traverse toutes les attitudes, les postures, les gestes des habitants du village, entre désir d'abolir la distance (de parvenir au château) et désir de la maintenir (c'est parfois la position de K. lui-même), et entre les deux toute une escorte d'intermédiaires, messagers, secrétaires, fonctionnaires, et tout l'espace de l'imaginaire et du fantasme, qui exclut toute possibilité d'un accueil vrai.

Pourtant, dans les toutes dernières lignes du texte inachevé, au chapitre 25, quelque chose se passe qui ressemble à de l'hospitalité. On y retrouve un personnage nommé Gerstäcker, que l'on avait rencontré au début du roman : c'est lui qui, désigné alors comme "voiturier", avait amené $\mathrm{K}$. à l'auberge. Cette première rencontre ${ }^{11}$ l'avait montré sous un jour plutôt antipathique. Mais les dernières lignes sont plus ambiguës. Gerstäcker invite K. à le suivre et à s'installer chez lui ; K., dans un premier temps, refuse, et toutes les raisons invoquées alors pour le convaincre sont aussi peu crédibles les unes que les autres; Gerstäcker promet gîte, couvert et même un salaire à K., dit qu'il a besoin de lui, mais n'explique pas en quoi consiste ce besoin. La discussion se termine sur une sorte de boutade, après quoi K., on ne sait pas vraiment pourquoi, finit par accepter de suivre Gerstäcker. Les dernières phrases du Château de Kafka sont les suivantes :

K. se mit à rire, s'accrocha à son bras et se laissa conduire dans la nuit.

La cabane de Gerstäcker était pauvrement éclairée par le feu de la cheminée et par un bout de bougie à la lueur de laquelle quelqu'un lisait dans une niche, sous les poutres obliques du toit qui faisaient saillie [à cet endroit]. C'était la mère de Gerstäcker. Elle tendit à K. une main tremblante et le fit s'asseoir à ses côtés. Elle parlait difficilement, on avait peine à la comprendre, mais ce qu'elle disait... ${ }^{12}$

Le texte s'interrompt sur les mots "mais ce qu'elle disait »; on ne saura jamais ce qu'elle disait, de même que restera à jamais indécidable le geste de Gerstäcker : relèvet-il d'une hospitalité généreuse, ou plutôt d'un intérêt bien compris? Le roman se termine certes par l'évocation d'un intérieur accueillant, d'une personne apparemment bienveillante (la mère), d'un rapport au texte (elle lit) et à la parole (elle parle, même si l'interruption définitive du roman laisse dans l'ombre la teneur de ses paroles). Mais le texte de Kafka n'accorde pas davantage de contenu explicite à cette hospitalité relative des Gerstäcker qu'aux actions et postures qui la précèdent. En outre, cet ultime épisode occupe dans Le Château une position inverse de celui de l'hospitalité des Phéaciens : là où celle-ci engageait le récit, celle-là au contraire fixe le texte sur son inachèvement, sur son silence définitif.

21 Le Château de Kafka est un univers dans lequel le tiers s'est éloigné ou a disparu, mais dans lequel demeure son lieu: un espace vide. D'où la nécessité d'inventer des intercesseurs, messagers, passeurs, voituriers, fonctionnaires de toutes sortes, euxmêmes partagés entre la futilité, l'arbitraire et l'imposture : ils doivent faire comme si le lien était encore réel et parfois ils le croient eux-mêmes, mais en réalité ils sont forcément dans l'erreur et dans le mensonge quand ils prétendent combler le vide alors 
qu'ils ne devraient que témoigner de la distance incommensurable qui les tient sous son joug.

K. au village, c'est en quelque sorte Ulysse chez les Phéaciens quand le tiers nécessaire à l'hospitalité manque. Le contrat, la convention sont omniprésents, mais en creux, par défaut: toutes les discussions entre les villageois et $\mathrm{K}$. tournent autour d'un hypothétique rapport conventionnel entre les choses du château et les choses du village, rapport jamais démenti, toujours au contraire expressément attesté, mais dont l'explicitation des termes effectifs est toujours ajournée.

Entre Homère et Kafka, il s'est produit à la fois un retournement et un évidement du schème de l'hospitalité : le contrat demeure, mais il est maintenant vide; il ne peut donc plus y avoir de réciprocité. L'univers de Kafka est un monde dans lequel seule la nécessité de la compensation demeure, alors que le don explicite qui rendait cette compensation nécessaire et lui donnait sens n'a plus de réalité.

Il serait pourtant très excessif de réduire la littérature européenne à une telle histoire de retournement et d'évidement. Car elle est aussi travaillée, en parallèle, par un fantasme qui ne se contente pas d'inquiéter le schème, mais qui voudrait s'en émanciper, au nom d'une immédiateté qui excéderait, au moins en apparence, la dimension contractuelle: une immédiateté positive, si l'on veut, contrairement à ce que nous trouvions chez Homère, une immédiateté au-delà de toute xenía, un accueil non institutionnel, une compensation sans convention.

À titre d'exemple, voici une version hyperboliquement «chrétienne » et une version « juive » de ce désir d'immédiateté.

La version chrétienne, je la trouve dans la reprise par Flaubert, dans ses Trois contes de 1877, de la légende de saint Julien l'Hospitalier. L'histoire était racontée dans la Légende dorée de Jacques de Voragine, dans les années 1260 , et à la même période, dans le Speculum historiale de Vincent de Beauvais. Il y est aussi fait allusion dans le deuxième récit de la deuxième journée du Decameron de Boccace écrit entre 1349 et 1353, et on en trouve encore une mention dans le Catalogus Sanctorum de Petrus de Natalibus écrit à la fin du XIV ou au début du Xv siècle : " parce qu'il fut l'hôte des pauvres et des pèlerins, les voyageurs l'invoquent pour trouver bon gite sous le nom de Julien l'Hospitalier. »

Dans cette tradition, le récit est, globalement, le suivant: Julien est un jeune homme noble. Il va à la chasse dans son vaste domaine et un jour, alors qu'il est sur le point d'abattre un cerf, celui-ci se retourne et lui dit: "Pourquoi me poursuis-tu, toi qui seras meurtrier de ton père et de ta mère ? " Julien est effrayé par ces paroles, et pour éviter que se réalise la prédiction du cerf il quitte sa famille et son domaine, s'enfuit dans une région très lointaine où il se met au service d'un prince qui finit par le faire chevalier et lui donne pour femme une noble veuve châtelaine qui lui apporte en dot son château.

Pendant ce temps-là, les parents de Julien, éplorés, se mettent à sa recherche. Un jour, ils parviennent à la bonne adresse, mais Julien n'est pas là ; ils sont accueillis par sa femme qui les reçoit avec honneur et leur propose son propre lit pour y dormir. Pendant la nuit, Julien rentre, se rend compte que le lit est occupé, croit que sa femme est couchée là avec un amant et les égorge l'un et l'autre, crime qui accomplit la prédiction du cerf. Nouvelle fuite, désir de repentance : Julien et sa femme quittent le pays et construisent, près d'un fleuve très difficile à traverser, un grand hôpital. Et ils 
restent là pendant tout le temps de la pénitence, servant de passeurs à ceux qui veulent traverser le fleuve et donnant l'hospitalité aux pauvres.

$\mathrm{Au}$ milieu d'une nuit très froide, Julien entend une voix implorante. Il fait passer le fleuve à un inconnu transi de froid, l'accueille dans sa maison, allume du feu pour le réchauffer et le fait coucher dans son lit sous ses propres couvertures. Et un peu après, l'inconnu, dont la première apparence était celle d'un homme faible, transi, " comme lépreux ", se transfigure en ange, devient rayonnant et s'élève vers le ciel en disant à son hôte : «Julien, le Seigneur m'a envoyé vers toi pour te montrer qu'il a accepté ta pénitence et dans peu de temps vous reposerez tous deux dans le Seigneur. » Et de fait, quelque temps plus tard, Julien et sa femme meurent dans la paix du Seigneur.

Dans l'ensemble de son texte, Flaubert suit assez fidèlement la légende. Mais il y ajoute principalement deux choses. D'abord il fait de Julien jeune un chasseur cruel, tuant arbitrairement pour le plaisir de tuer, sanguinaire, par moments submergé par une irrépressible pulsion de meurtre ; ce faisant, Flaubert ajoute en quelque sorte à la lutte de Julien contre le destin celle qu'il doit mener contre ses propres pulsions meurtrières. Du coup, sa repentance, qui prend la forme de l'hospitalité, acquiert une signification nouvelle : elle est aussi une façon de compenser un désir charnel, une violence inscrite dans la chair même du personnage.

31 La deuxième grande nouveauté chez Flaubert, c'est la fin. À la visitation angélique, il substitue un accouplement charnel, une sorte de scène d'amour physique au terme de laquelle le personnage accueilli, un lépreux, entraîne Julien dans la mort comme dans un paroxysme de jouissance. Julien fait traverser le fleuve au lépreux, lui donne à manger et à boire tout ce qu'il possède, puis l'installe dans son propre lit. Le lépreux lui demande alors :

- Déshabille-toi, pour que j'aie la chaleur de ton corps!

Dans les dernières phrases de la nouvelle, on voit Julien se dénuder, serrer contre lui le lépreux, le chevaucher " complètement, bouche contre bouche, poitrine sur poitrine ", et Flaubert décrit précisément la "clarté d'étoile » des yeux du lépreux, les " rais du soleil» qu'évoquent ses cheveux, la «douceur des roses» de son souffle. Le texte se termine par le paragraphe suivant :

Cependant une abondance de délices, une joie surhumaine descendait comme une inondation dans l'âme de Julien pâmé; et celui dont les bras le serraient toujours grandissait, grandissait, touchant de sa tête et de ses pieds les deux murs de la cabane. Le toit s'envola, le firmament se déployait; - et Julien monta vers les espaces bleus, face à face avec Notre Seigneur Jésus, qui l'emportait dans le ciel ${ }^{13}$.

La caractéristique spécifiquement chrétienne de cette version n'est pas seulement attestée dans cette dernière ligne qui nomme expressément « Notre Seigneur Jésus »: elle tient surtout à ce que l'hospitalité y rachète non seulement une faute commise par erreur, mais surtout un péché inscrit dans la chair même. C'est pourquoi la rédemption ne peut être elle-même qu'un acte de chair. La sainteté de l'hospitalier ne consiste pas en une référence à un tiers quelconque, dieu présent ou lieu vide du divin, elle n'a pas davantage de motivation institutionnelle ou juridique - ici, pas de contrat, de convention: toute l'histoire est encadrée par deux événements qui inscrivent Julien dans la loi, positivement et négativement - la prédiction initiale du cerf et l'acte christique du salut -, mais aucun de ces actes n'a de teneur contractuelle. Entre les deux, Julien s'efforce d'échapper à son destin et à ses pulsions, tente de compenser le péché par la fuite hors de lui-même, puis par l'hospitalité, mais en quelque sorte sans 
intercesseur. Entre Julien et ses pulsions meurtrières, puis entre Julien et sa sainteté, il n'y a rien, personne. L'hospitalité est une compensation, mais dans l'immédiateté, dans l'absence de médiation.

Elle en est d'autant plus ambivalente, puisqu'elle est en quelque sorte l'envers immédiat de la faute. Flaubert dessine ici la figure saisissante, pathétique, d'une compensation omniprésente, mais qui ne donne lieu à aucun contrat ou aucune convention explicite, une compensation immédiatement charnelle.

On retrouve une sorte de dimension d'immédiateté, mais évidemment très différente, sinon opposée dans son inspiration, dans un épisode raconté par Edmond Jabès dans un ouvrage qui s'intitule précisément Le Livre de l'hospitalité, paru en $1991^{14}$. Dans l'écriture et la poésie d'Edmond Jabès, son origine de Juif égyptien est déterminante, sans cesse évoquée, parfois de manière biographique, parfois plutôt par le biais des thématiques abordées : le désert, la loi, la distance. Jabès est né en 1912, il a vécu en Égypte jusqu’à son installation à Paris en 1956. Il est mort à Paris en 1991. Dans Le Livre de l'hospitalité, qui est l'un de ses tout derniers écrits et le dernier livre publié de son vivant, il raconte un début de voyage. Il est jeune, accompagné de quelqu'un qui n'est pas nommé dans le texte, mais auquel il s'adresse par un tutoiement. Ils traversent le canal de Suez, s'engagent dans le désert du Sinaï. Mais une fausse manœuvre fait que leur voiture se renverse, que leurs réserves d'eau se brisent, qu'ils ne peuvent plus avancer et qu'ils ne savent pas où aller s'ils rebroussent chemin à pied. Dans la nuit, ils entendent une voix et se trouvent face à un nomade qui les interroge, auquel ils racontent leur mésaventure et qui les aide spontanément en disant, - "peut-être, écrit Jabès, pour nous mettre à l'aise " - : " “N'êtes-vous pas mes hôtes ?" ". Jabès continue ainsi : " Nous ne savions pas très bien comment lui témoigner notre reconnaissance. / Tu lui offris un peu d'argent qu'il refusa, offusqué. / Ta maladresse était, à ses yeux, si énorme qu'elle ne pouvait être qu'innocente. Il nous tendit, en souriant, la main et s'éclipsa. »

La suite est racontée par Jabès en ces termes : les voyageurs reviennent quelques jours plus tard au même endroit, le même nomade s'approche d'eux et les accueille, mais il le fait comme s'il ne les connaissait pas. Ils sont choqués par cette attitude, dont ils ne voient pas la raison. Puis ils réfléchissent et en viennent finalement à comprendre ce qu'il en est de « l'hospitalité des Bédouins » :

Si notre hôte nous avait reçus en feignant de nous ignorer, c'était pour marquer que nous restions, l'un et l'autre, à ses yeux, les anonymes voyageurs qu'il lui fallait, au nom de l'ancestrale hospitalité de sa tribu, honorer en tant que tels car, autrement, notre visite improvisée aurait, rapidement, fait figure d'éphémères retrouvailles ${ }^{15}$.

37 Jabès donne à l'épisode le sens d'une sorte de leçon à méditer, comme si ces deux Juifs accueillis par un Bédouin recevaient de lui un enseignement qui, pour Jabès, engage aussi la parole et l'éthique du judaïsme. La leçon tient dans l'opposition finale entre retrouvailles et hospitalité. Les retrouvailles seraient une répétition du déjà connu, une réitération de l'identique, du même : une reconnaissance, un acte à ce titre intégré dans l'ordre du savoir, c'est-à-dire aussi du pouvoir. Mais il faut - c'est la loi, la paradoxale loi de l'immédiateté - que l'hospitalité échappe à l'ordre de la connaissance, qu'elle l'excède infiniment, qu'elle soit accueil de l'étranger au sens de celui qu'on ne connait pas, qui n'a pas de nom, qui est autre infiniment. Bien sûr, Jabès a auparavant indiqué que "l'hospitalité particulière aux natifs du désert" - qui fait que "celui qui, inopinément, se présente à vous a, toujours, sa place réservée sous la tente »-, tient à ce que cet arrivant inopiné « est l'envoyé de Dieu ». Mais cette idée n'est pas réductible 
à ce que nous trouvions chez Homère, parce que précisément ici le Dieu qui envoie non seulement ne se manifeste pas comme tel, mais est l'imprésentable même. De sorte que pour que l'étranger soit "l'envoyé de Dieu », il faut qu'il soit anonyme, non connu et reconnu, qu'il porte en quelque sorte sur lui-même des traces de l'imprésentable. L'idée est donc: "Accueillir autrui pour sa seule présence, au nom de sa seule existence, uniquement pour ce qu'il représente. / Pour ce qu'il est ${ }^{16}$.»

Et pourtant, si l'hospitalité ne se réduit pas à la convention, à la définition, à la circonscription, elle n'est pas non plus sans loi. Bien au contraire. Mais la loi à laquelle elle obéit n'est pas une loi de la compensation, de l'égalisation, de l'équivalence ou de la réciprocité, c'est une loi de la dissymétrie, de la distance, de l'excédent.

Dans le même livre, Jabès relate le dialogue suivant: «- Quelle définition pourrait convenir à l'hospitalité ? - demanda, à son maître, le plus jeune de ses disciples. / Une définition est, en soi, une restriction et l'hospitalité ne souffre aucune limitation répondit le maître ${ }^{17}$. » Cette réponse place l'hospitalité au-delà de toute circonscription, elle lui accorde une extension illimitée, celle du désert. Mais le fait que "l'hospitalité ne souffre aucune limitation » n'est pas un constat, c'est bien une loi. Une loi au-delà de toute législation explicite, de toute figuration, de toute reconnaissance, de toute convention. L'hospitalité n'est pas une affaire de réciprocité, mais de partage de l'infini inassignable. C'est pourquoi elle a, pour Jabès, à voir avec l'attente - « Hospitalière est, par-dessus tout, l'attente ${ }^{18}$ »-, avec la disponibilité - «La totale disponibilité débouche sur l'hospitalité ${ }^{19}$ »-, et finalement avec le Rien qui est aussi le commencement de l'écriture : "Ô forces déployées d'un indicible futur. / Le Rien est prémices. / Tolérante absence. Originelle hospitalitée ${ }^{20}$ "; "Ainsi, la "chose à transmettre" n'était que poussière de sable et le livre, poussière de vocables. / Tout est à réécrire. / Naissance de l'hospitalitée ${ }^{11}$. " Le langage lui-même est hospitalité, quand il n'est pas transmission de message, instrument de communication, mais chemin, ouverture, fêlure d'infinité dans le fini, intervalle.

Je voudrais terminer par une question, une question qui, je le crains, demeurera sans réponse, et une allusion à deux philosophes qui, s'ils ne nous permettent pas d'y répondre, pourront au moins nous aider à construire cette question. Ces deux philosophes sont Emmanuel Levinas et Jacques Derrida. La question, elle, est celle-ci : nous, nous Européens d'aujourd'hui, où en sommes-nous avec cette notion d'hospitalité, nous qui chaque jour sommes informés de situations dramatiques de réfugiés en Méditerranée et sur les frontières de l'Europe, nous qui nous préoccupons enfin d'accueillir à peu près décemment ceux qui comme Ulysse, parfois avec beaucoup moins de chance, abordent nos côtes, pour y survivre ou pour y mourir, nous qui chaque jour côtoyons dans nos villes des "sans domicile fixe", nous qui, pour cette raison et pour bien d'autres, sentons la nécessité d'une pensée de l'hospitalité qui corresponde à notre temps?

41 Le présupposé de cette question, c'est que notre situation ne serait plus pensable dans les termes qui étaient ceux des œuvres littéraires que nous venons de citer. Nous n'aurions plus d'équivalent de cette xenía effective dont dépend, dans l'odyssée, l'accueil de l'étranger. Nous n'aurions plus non plus cette Loi du partage de l'infini qui fait de l'étranger une voix et un pas interrompant le désert, nous ne croyons plus vraiment à une immédiate rémission charnelle des péchés, et si finalement nous ressemblons davantage aux villageois du Château pour lesquels la xenía est d'autant plus formellement contraignante qu'elle est désormais vidée de tout contenu, il est probable 
que nous nous soyons émancipés aussi de cette nécessité de la loi absurde qui chez Kafka avait au moins l'avantage de maintenir quelque chose comme l'idée intraitable de la distance et de la séparation, c'est-à-dire aussi de l'étrange ou de l'étranger. prise et même de toute compréhension. C'est cette altérité infinie qu'il s'agissait, selon lui, d'accueillir. L'hospitalité, pensait-il, n'est qu'un mot, elle n'est pas un acte éthique, si elle n'est pas l'accueil en l'autre de ce qui, en lui, est absolument irréductible, de son visage. Derrida appelle cela l'hospitalité «inconditionnelle»: sans condition, sans institution, au-delà de toute réciprocité, de tout contrat, de toute équivalence; une relation absolument dissymétrique, qui ne réduit pas la séparation et la distance mais au contraire les atteste, relation dans laquelle je n'octroie rien, aucun droit, à l'autre, parce qu'il est, dans sa finitude, le transcendant pur, au-delà de toute égalité et de toute compensation. La loi éthique de l'hospitalité est exclusive de tout pouvoir et de toute possession, y compris la possession de la maison dans laquelle l'autre est accueilli, maison dont Levinas écrit qu'elle est déjà elle-même « hospitalière à son propriétaire ${ }^{22}$ ", lequel est donc toujours précédé par un don ou un appel immémorial sur lequel il n'a pas de prise, dont il ne décide pas lui-même. La loi éthique de l'hospitalité inconditionnelle commande la réponse à la question que Levinas formule ainsi :

Abriter l'autre homme chez soi, tolérer la présence des sans-terre et des sans-

domicile sur un « sol ancestral » si jalousement - si méchamment - aimé, est-ce le critère de l'humain? Sans conteste ${ }^{23}$.

Mais on trouve aussi chez Levinas une autre dimension de la relation. Le face-à-face éthique, l'exclusivisme de l'apparaître du visage, l'ouverture à la transcendance de l'autre se trouvent tempérés ou complétés par ce que Levinas appelle le tiers, le Nous, la forme de l'universalité : nous quittons alors le plan de l'éthique dans son exigence métaphysique d'infini et d'inconditionnalité et retrouvons la dimension de l'institution, de la législation, de la convention. Cela est inévitable, par le simple fait que nous vivons en société, mais cela comporte aussi le risque d'une altération de la relation éthique, de sa déformation, d'une soumission excessive et du moi et de l'Autre à une identification dominatrice et potentiellement tyrannique ${ }^{24}$.

Nous sommes donc dans la difficulté suivante : l'éthique, la loi de l'éthique nous enjoint une hospitalité absolue, inconditionnelle, sans calcul, sans tri, sans exercice du pouvoir, sans compromis, sans discussion; et le droit, la politique, le sens social de la justice ne peuvent que contredire cette loi au nom de l'institution, de son fonctionnement, de sa propre logique. Nous sommes dans le conflit de deux lois, comme dans la tragédie grecque. Et comme dans la tragédie grecque, ces deux lois s'opposent et chacune a sa légitimité.

Jacques Derrida parle à ce propos $\mathrm{d}^{\prime}$ " antinomie ${ }^{25}$ ». Il y a, dit-il, pour nous, une contradiction insoluble entre la loi de l'hospitalité absolue et de l'accueil sans condition et les lois de l'hospitalité comme droit ou devoir, comme "pacte ». La première « exige que j'ouvre mon chez-moi et que je donne non seulement à l'étranger (pourvu d'un nom de famille, d'un statut social d'étranger, etc.) mais à l'autre absolu, inconnu, anonyme, et que je lui donne lieu, que je le laisse venir, que je le laisse arriver, et avoir lieu dans ce lieu que je lui offre, sans lui demander ni réciprocité (l'entrée dans un pacte) ni même son nom. » Mais les secondes, les lois institutionnelles de l'hospitalité, 
régissent ce que Derrida appelle «l'hospitalité de droit ", « la loi ou la justice comme droit », elles fixent « les conditions, les normes, les droits et les devoirs qui s'imposent aux hôtes et aux hôtesses, à ceux ou à celles qui donnent comme à ceux ou à celles qui reçoivent l'accueil ${ }^{26}$.»

D’un côté, donc, la "loi inconditionnelle de l'hospitalité illimitée ", qui consiste à « donner à l'arrivant tout son chez-soi et son soi, lui donner son propre, notre propre, sans lui demander ni son nom, ni contrepartie, ni de remplir la moindre condition », de l'autre «les lois de l'hospitalité, ces droits et ces devoirs toujours conditionnés et conditionnels, tels que les définit la tradition gréco-latine, voire judéo-chrétienne ${ }^{27}$ »et pas de coïncidence, d'adéquation, pas d'équivalence, pas de conciliation entre les deux. Une antinomie insoluble, là où, la littérature européenne en témoigne, toute notre histoire, depuis les rédactions à peu près contemporaines des Chants d'Homère et des premiers écrits de la Thora, aura tenté, en accusant les ambivalences, d'accorder entre elles la loi de la transcendance et les lois de la contingence.

Que faire alors, si notre lot est celui de circuler tant bien que mal dans l'espace tendu, instable, insatisfaisant de l'antinomie? Cela inspire des attitudes nettement contrastées: la violence de l'imposition autoritaire d'une Loi censée reconnecter la contingence et l'absolu (c'est le sens des intégrismes et fondamentalismes); ou le désespoir de ne pouvoir supporter la distance, la faille, l'inadéquation entre le fini et l'infini, la non-coïncidence entre convention et transcendance ; ou encore toutes sortes de cynismes et d'hypocrisies difficilement évitables et abondamment confirmées de nos jours.

49 Pour Jacques Derrida, la situation aporétique, « loin de paralyser le désir ou de détruire l'exigence d'hospitalité », a plutôt le sens d'un devoir qui nous serait imposé. Elle exige de nous que nous établissions ce qu'il appelle des "schèmes intermédiaires » entre l'inconditionnalité d'une loi et d'un désir absolus d'un côté, et de l'autre le caractère conditionnel des règles juridiques et politiques qui dans une société de droit régissent effectivement et concrètement l'accueil. Cette sommation est en grande partie " impossible ", dans la mesure où les termes d'une «négociation » entre l'absolu et le relatif, l'incommensurable et le calculable ne nous sont pas connus. Et c'est pourtant ce à quoi Derrida nous invite. Mais cette invitation n'a pas le sens d'un « juste milieu », ni d'une synthèse formelle. Elle vise à la constitution d'un droit qui soit à la fois, en tant que tel, "déterminé, limitable et délimitable " et qui cependant réponde aux « injonctions nouvelles de situations historiques inédites » et, les intégrant, satisfasse aussi aux exigences non conditionnelles. Mais à cet endroit, le texte semble ne pouvoir que multiplier les questions : "Comment donner lieu à une politique et à une éthique concrètes ", qui aille dans ce sens ? De quelle manière y parvenir : " en changeant les lois, en déterminant autrement la citoyenneté, la démocratie, le droit international, etc. ? En intervenant donc réellement dans la condition de l'hospitalité au nom de l'inconditionnel ${ }^{28}$ ?"

Je ne peux, malheureusement, faire mieux qu'en rester à ces mêmes questions. 


\section{BIBLIOGRAPHIE}

Benveniste Émile, Le Vocabulaire des institutions indo-européennes, Paris, Éditions de Minuit, coll. « Le Sens commun », 1969.

Derrida Jacques et Anne Dufourmentelle, De l'hospitalité, Anne Dufourmentelle invite Jacques Derrida à répondre, Paris, Calmann-Lévy, coll. « Petite bibliothèque des idées », 1997.

Derrida Jacques, Adieu à Emmanuel Levinas, Paris, Galilée, coll. « Incises », 1997.

Flaubert Gustave, Trois Contes, Préface de Michel Tournier, édition établie et annotée par S. de Sacy, Paris, Gallimard, coll. « Folio », 1973.

Homère, Odyssée, Chant VI, traduction Victor Bérard, Introduction et notes Jean Bérard, Paris, Le Livre de poche, 1960.

Jabès Edmond, Le Livre de l'hospitalité, Paris, Gallimard, 1991.

Kafka Franz, « Le Château », traduction Alexandre Vialatte, Euvres complètes, tome 1, Paris, Gallimard, coll. « Bibliothèque de la Pléiade », 1976.

Levinas Emmanuel, À l'heure des nations, Paris, Éditions de Minuit, coll. « Critique », 1988.

Levinas Emmanuel, Totalité et Infini, La Haye, Martinus Nijhoff, 1961.

\section{NOTES}

1. Benveniste Émile, Le Vocabulaire des institutions indo-européennes, Paris, Éditions de Minuit, 1969,

t. 1, p. 94-96.

2. Benveniste, 1969, p. 92.

3. Ibid., p. 93-94.

4. Ibid., p. 94.

5. Id.

6. Homère, Odyssée, Chant VI, traduction Jean Bérard, Paris, Le Livre de poche, 1960, p. 125 et 128.

7. Ibid., p. 130-131.

8. Ibid., p. 136.

9. Ibid., p. 141.

10. Kafka Franz, Euvres complètes, traduction Alexandre Vialatte, Paris, Gallimard, coll. « Bibliothèque de la Pléiade », t. 1, 1976, p. 504.

11. Ibid., p. 507-509.

12. Ibid., p. 808.

13. Flaubert Gustave, Trois contes, Paris, Gallimard, coll. « Folio », 1973, p. 97.

14. Jabès Edmond, Le Livre de l'hospitalité, Paris, Gallimard, 1991, p. 81-85.

15. Ibid., p. 84-85.

16. Ibid., p. 21.

17. Ibid., p. 57.

18. Ibid., p. 22.

19. Ibid., p. 67.

20. Ibid., p. 71-72.

21. Ibid., p. 96.

22. Levinas Emmanuel, Totalité et Infini, La Haye, Martinus Nijhoff, 1961, p. 131. 
23. Levinas Emmanuel, À l'heure des nations, Paris, Éditions de Minuit, 1988, p. 114.

24. Levinas Emmanuel, Totalité et Infini, op. cit., p. 276 : «[...] la politique laissée à elle-même, porte en elle une tyrannie. Elle déforme le moi et l'Autre qui l'ont suscitée, car elle les juge selon les règles universelles et, par là même, comme par contumace ".

25. Je me réfère ici à deux textes dans lesquels Derrida traite directement de la question de l'hospitalité : Jacques Derrida, Adieu à Emmanuel Levinas, Paris, Galilée, 1997 ; Jacques Derrida et Anne Dufourmentelle, De l'hospitalité, Paris, Calmann-Lévy, 1997.

26. Derrida Jacques, De l'hospitalité, op. cit., p. 29.

27. Ibid., p. 73.

28. Ibid., p. 129-131.

\section{RÉSUMÉS}

Comment la littérature européenne traite-t-elle la question de l'hospitalité ? Confrontée aujourd'hui à ses frontières et, sur ses côtes, à la demande d'accueil de nombreux réfugiés et «migrants", l'Europe peut-elle espérer trouver dans sa littérature de quoi se ressaisir et retrouver la voie d'une authentique disponibilité à leur égard, au-delà de l'alternative piégée de l'exclusion violente et de la compassion impuissante?

La leçon d'Homère racontant la bonne fortune d'Ulysse accueilli par les Phéacéens semble totalement oubliée, dans Le Château de Kafka, par les habitants du village qui menacent $\mathrm{K}$. dès son arrivée, avec des mots que malheureusement nous ne sommes plus surpris d'entendre aujourd'hui braillés par des Européens : «l'hospitalité n'est pas d'usage chez nous, nous n'avons pas besoin d'hôtes "!

Contrairement aux idéologies et aux simplifications dogmatiques, la littérature arpente l'espace inévitablement tendu et aporétique qui s'étend, à propos de l'hospitalité, entre une loi inconditionnelle d'accueil et des lois circonstancielles relatives aux temps et aux lieux.

\section{INDEX}

Mots-clés : hospitalité, littérature, étranger, éthique, loi, institution

\section{AUTEUR}

\section{DANIEL PAYOT}

Daniel Payot est professeur à l'Université de Strasbourg et membre de l'unité de recherche ACCRA - Approches contemporaines de la création et de la réflexion artistiques. 